Document downloaded from:

http://hdl.handle.net/10251/99754

This paper must be cited as:

Conejero, JA.; Martínez Jiménez, F.; Peris Manguillot, A.; Ródenas Escribá, FDA. (2016). Chaotic asymptotic behaviour of the solutions of the Lighthill Whitham Richards equation. Nonlinear Dynamics. 84(1):127-133. doi:10.1007/s11071-015-2245-4

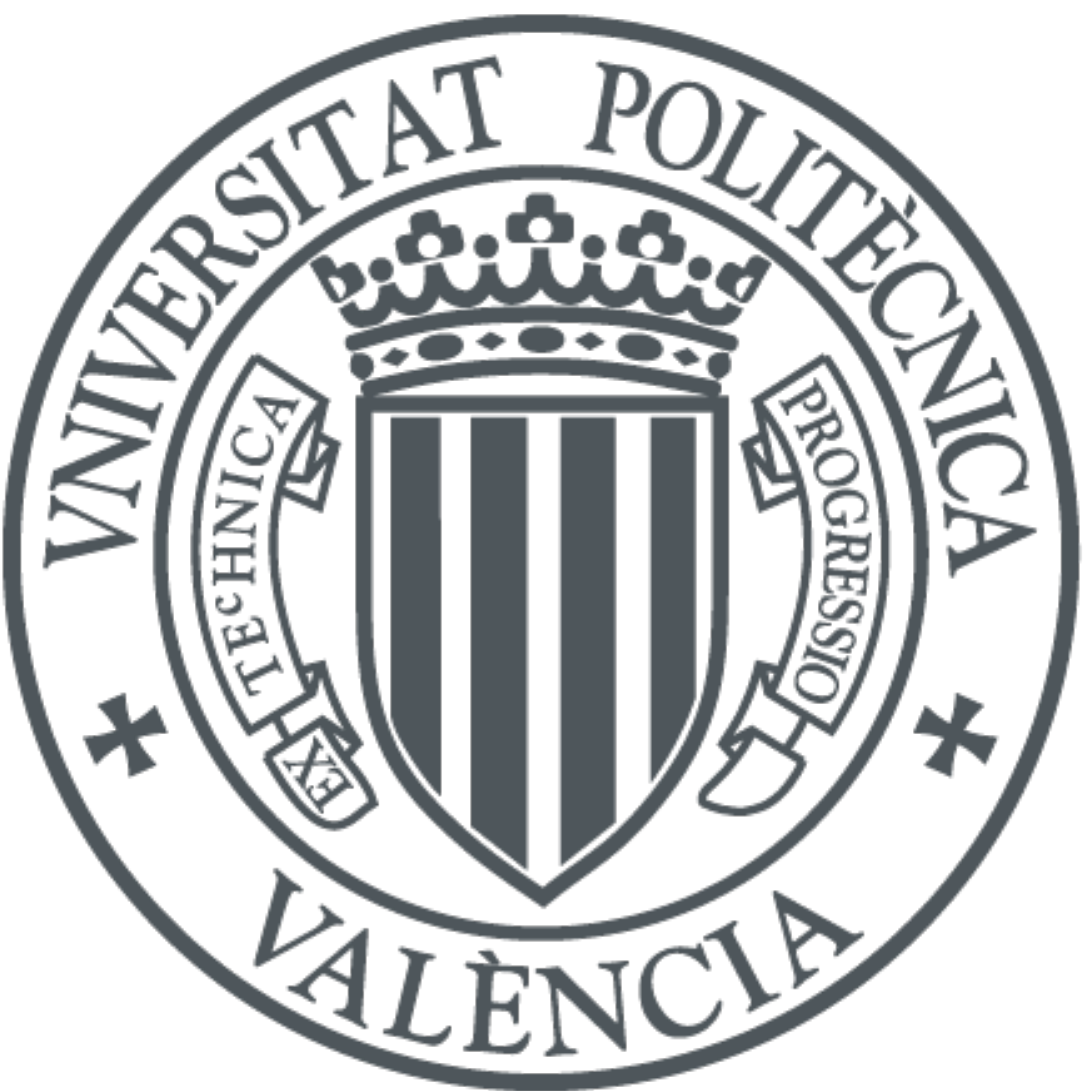

The final publication is available at

http://doi.org/10.1007/s11071-015-2245-4

Copyright Springer-Verlag

Additional Information 


\title{
Chaotic asymptotic behaviour of the solutions of the Lighthill-Whitham-Richards equation
}

\author{
J. Alberto Conejero, Félix Martínez-Giménez, Alfredo Peris, Francisco Ródenas \\ Instituto Universitario de Matemática Pura y Aplicada, Universitat Politècnica de València, València, Spain
}

\begin{abstract}
The phenomenon of chaos has been exhibited in mathematical nonlinear models that describe traffic flows, see for instance Li05, LG04. At microscopic level, Devaney chaos and distributional chaos has been exhibited for some car-following models, such as the Quick-Thinking-Driver model and the Forward and Backward control model CMASS15, BCMASS15. We present here the existence of chaos for the macroscopic model given by the Lighthill-Whitmam-Richards equation.
\end{abstract}

Keywords: Devaney chaos; mixing measures; traffic; Lighthill-Whitman-Richards equation; $C_{0}$-semigroup. 2010 MSC: 47A16.

\section{Introduction}

Chaos is usually identified with nonlinear and unpredictable phenomena. However, chaotic dynamics can be also found in dynamical systems described by some examples linear and continuous operators. This can only happen if the underlying space was infinite-dimensional. In addition, this can be exhibited in process whose evolution is described in a deterministic way in terms of differential equations.

For the common man, one of the settings where chaos can appear is in traffic. Mathematical models for describing traffic processes can be found at microscopic and macroscopic level. On the one hand, at microscopic level every single vehicle is simulated, describing its position and velocity. At this level we can find car-following models, which depend continuously respect to the time, and intend to represent the traffic in terms of ordinary differential equations. More information on these models can be found in BM99

We can also find cell automaton models that are based on a discretization of the time and the space. Then the dynamical system describes if we can find a car or not at each cell, see [MDM05. On the other hand, at macroscopic level we find traffic flow models that intend to integrate the single behaviour of each vehicle and to compute some characteristics of the system as a whole.

In the linear setting, chaos has also been found at microscopic level for some car-following traffic models. These models intend to describe the behaviour of each car in terms of the difference of its speed respect to the speed of the cars near of it. In addition, the reaction time of the drivers is also taking into the description of the model. Once we represent the solutions to a given model as a $C_{0}$-semigroup, then there exists certain initial conditions of the speeds of the cars that exhibit a chaotic behaviour. More precisely, the infinite version of the Quick-Thinking-Driver (IQTD) model has been introduced in CMASS15. Let $\left(u_{i}(t)\right)_{i}$ denote the speeds of an infinite number of cars on a track. Suppose that for every $i \in \mathbb{N}$, the car $i$

\footnotetext{
* Corresponding author

Email addresses: aconejero@upv.es (J. Alberto Conejero), fmartinez@mat.upv.es (Félix Martínez-Giménez), aperis@mat.upv.es (Alfredo Peris), frodenas@mat.upv.es (Francisco Ródenas)

$U R L$ : albertoconejero.webs.upv.es (J. Alberto Conejero), personales.upv.es/aperis (Alfredo Peris)
} 
behaves following the (QTD) model respect to one in front of it, the car $i+1$. The following infinite system of ordinary differential equations represents this situation:

$$
u_{i}^{\prime}(t)=\lambda_{i}\left(u_{i+1}(t)-u_{i}(t)\right) \text { for } i \in \mathbb{N} .
$$

When considering the speeds of the cars in $\ell_{1}(s), 0<s<1$, the weighted space of summable sequences defined as

$$
\ell_{1}(s)=\left\{\left(v_{i}\right)_{i \in \mathbb{N}} \in \mathbb{K}^{\mathbb{N}}:\left\|\left(v_{i}\right)_{i \in \mathbb{N}}\right\|_{s}=\sum_{i \in \mathbb{N}}\left|v_{i}\right| s^{i}<\infty\right\}
$$

can be considered to pose the following abstract Cauchy problem on it:

$$
\left\{\begin{array}{l}
u^{\prime}(t)=A u(t) \\
u(0)=\left(u_{i}(0)\right)_{i \in \mathbb{N}}
\end{array}\right\}
$$

for $u=\left(u_{i}(t)\right)_{i \in \mathbb{N}} \in \ell_{1}(s)$ and $t \geq 0$, being $\left(u_{i}(0)\right)_{i \in \mathbb{N}}$ the vector of speeds of the cars at $t=0$. Then, the solutions to this problem can be analyzed from the point of view of $C_{0}$-semigroups. Further information on $C_{0}$-semigroups will be provided in Section 2 .

In the (QTD) model the velocity of a car is only controlled respect to the speed of the car in front of it. However, it can be also controlled taking into account the speeds of the car in front and behind of it. Such a model is known as the Forward and Backward Control model. The infinite version of it, the (IFBC) model, has been introduced in BCMASS15. This model is given by the following system of infinite ordinary differential equations:

$$
\begin{aligned}
& u_{1}^{\prime}(t)=-\mu_{1} u_{1}(t)+\mu_{2}\left(u_{2}(t)-u_{1}(t)\right), \\
& u_{i}^{\prime}(t)=\mu_{1}\left(u_{i-1}(t)-u_{i}(t)\right)+\mu_{2}\left(u_{i+1}(t)-u_{i}(t)\right), \text { for all } i \geq 2,
\end{aligned}
$$

with control constants $\mu_{1}, \mu_{2}>0, \mu_{1}<\mu_{2}$. Again, taking this equations instead of the ones in (1), one can also posed an abstract Cauchy problem on $\ell^{1}(s)$, too, and study the chaotic behaviour of some of its solutions.

The study of chaos for linear kinetic models,as the one we have presented, was firstly introduced by Protopopescu and Azmy in PA92. There they only considered the case of constant coefficients. Later, Banasiak and Lachowicz started the study of Devaney chaos for the formulation of these models with variable coefficients. They also formulated these models as death and birth-and-death models of cell proliferation, see BL01] for the death model and BL02 for the birth-and-death one. Their results were later extended in AP12, GEPM11, BM11, considering also the topologically mixing property. Finally, the study of distributional chaos is also developed in CMASS15] and BCMASS15.

In this note we will show that linear chaos can also appear for traffic models stated at macroscopic level. The most elementary of these models was developed by Lighthill and Whitham [LW55, and Richards Ric56. This model is described by the following continuity equation:

$$
\frac{\partial \rho}{\partial t}+\frac{\partial q}{\partial x}=0
$$

where $q$ is the flow rate of traffic and $\rho$ is the traffic density. Taking into account the average speed $v$, the variables $q$ and $\rho$ verify that

$$
\rho(t, x)=v(t, x) q(t, x) \quad \text { for every } t \geq 0, x \in \mathbb{R} .
$$

The model in (5) is based on the assumption that the the number of cars is preserved along the track between any pair of points and times. They used this model for showing the existence of shock waves in traffic. More information can be found in [Lus10]. 
This model has some limitations: No inertial effects are considered (therefore the speeds of the vehicles are adapted instantaneously) and no diffusive term is included that would model how drivers look ahead to adjust their speed. Then Lighthill and Whitham proposed a second order lineal model [LW55]:

$$
\frac{\partial u}{\partial t}+c \frac{\partial u}{\partial x}+T \frac{\partial^{2} u}{\partial t^{2}}-D \frac{\partial^{2} u}{\partial x^{2}}=0
$$

Here, $T$ is the inertial time constant for speed variation, $c$ is the wave speed, and $D$ is the diffusion coefficient that shows how drivers respond to changes far away from their position. This equation can be compared with the Hyperbolic Heat Transfer equation (HHTE) on a rod:

$$
\tau \frac{\partial^{2} u}{\partial t^{2}}+\frac{\partial u}{\partial t}=\alpha \frac{\partial^{2} u}{\partial x^{2}}
$$

where $u$ is the temperature at every moment at each point of the rod, $\alpha$ is the thermal diffusivity, and

is the thermal relaxation time. The study of the chaotic behaviour of the solutions to this model has been developed in CPT10, GEPM11, even in the presence of internal sources CRT15, which is called the Bioheat equation (BE). In all of these cases, the chaotic dynamical behaviour is found in spaces of real analytic functions whose coefficients has some control at the $\infty$.

Previously, Herzog study the dynamics of the solutions to the Fourier Heat Transfer equation (FHTE) 65 on an infinite rod in the absence of internal heat sources. The underlying spaces considered to study the chaos of these three examples, (FHTE), (HHTE), and (BE), is the space of real analytic functions on $\mathbb{R}$ with exponential decay, endowed with the compact open topology Her97. This space has recently been considered to study the dynamics of the solution to the Moore-Gibson-Thompson equation CLR15.

The paper is organized as follows: In Section 2 we introduce the basic notions on $C_{0}$-semigroups and linear dynamics. The spaces of analytic functions of Herzog type will also be presented. The study of chaos and the existence of invariant strongly mixing measurs will be exposed in Section 3.

\section{Preliminaries}

We present some definitions and preliminary results that will be needed for proving the main results of the paper.

\section{1. $C_{0}$-semigroups}

Let $X$ be an infinite-dimensional Banach space. A family $\left\{T_{t}\right\}_{t>0}$ of linear and continuous operators on $X$ is said to be a $C_{0}$-semigroup if $T_{0}=I d, T_{t} T_{s}=T_{t+s}$ for all $t, s \geq 0$, and $\lim _{t \rightarrow s} T_{t} x=T_{s} x$ for all $x \in X$ and $s \geq 0$. Given a $C_{0}$-semigroup $\left\{T_{t}\right\}_{t \geq 0}$ on $X$, it can be shown that an operator defined by

$$
A x:=\lim _{t \rightarrow 0^{+}} \frac{T_{t} x-x}{t},
$$

exists on a dense subspace of $X$; denoted by $D(A)$. Then $(A, D(A))$ is called the (infinitesimal) generator so of the $C_{0}$-semigroup $\left\{T_{t}\right\}_{t \geq 0}$. If $D(A)=X$, then the $C_{0}$-semigroup can be rewritten as $\left\{e^{t A}\right\}_{t \geq 0}$. Such a semigroup is the corresponding solution $C_{0}$-semigroup of the abstract Cauchy problem

$$
\left\{\begin{array}{ll}
u^{\prime}(t, x) & =A u(t, x) \\
u(0, x) & =\varphi(x),
\end{array}\right\} .
$$

The solutions to this problem can be expressed as $u(t, x)=e^{t A} \varphi(x)$, where $\varphi(x) \in X$. Further information on $C_{0}$-semigroups can be found in [EN00, Paz83]. 


\subsection{Linear dynamics of $C_{0}$-semigroups}

Given a family of operators $\left\{T_{t}\right\}_{t} \geq 0$ we say that it is transitive if for every pair of non-void open sets $U, V \subset X$ there exists some $t>0$ such that $T_{t}(U) \cap V \neq \emptyset$. Furthermore, if there is some $t_{0}>0$ such that the condition $T_{t}(U) \cap V \neq \emptyset$ holds for every $t \geq t_{0}$ we say that it is topologically mixing. A $C_{0}$-semigroup is hypercyclic if there exists some $x \in X$ such that the set $\left\{T_{t} x: t \geq 0\right\}$ is dense in $X$. In this setting, transitivity coincides with hypercyclicity, but it is strictly weaker than topologically mixing [BBCP05].

We recall that an element $\in X$ is said to be a periodic point of $\left\{T_{t}\right\}_{t \geq 0}$ if there exists some $t_{0}>0$ such that $T_{t_{0}} x=x$. A $C_{0}$-semigroup $\left\{T_{t}\right\}_{t \geq 0}$ is said to be chaotic in the sense of Devaney if it is hypercyclic and there exists a dense set of periodic points in $X$. The following criterion let us prove the Devaney chaos for a $C_{0}$-semigroup. This result can be compared with the Desch-Schappacher-Webb Criterion [DSW97, Th 3.1], or any of its extensions [BM05, CM10.

Let $X^{*}$ denote the dual space of $X$ of linear and continuous functionals on $X$. We recall that by a weakly analytic function $f: U \rightarrow X$ on an open subset $U \subset \mathbb{C}$ we understand an $X$-valued function such that, for every $x^{*} \in X^{*}$, the complex valued function $z \mapsto\left\langle f(z), x^{*}\right\rangle$ is analytic on $U$. In the sequel, $J$ is a nonempty index set.

Theorem 2.1. (GEPM11, Theorem 7.30]) Let $X$ be a complex separable Banach space and $\left\{T_{t}\right\}_{t \geq 0} a C_{0}$ semigroup on $X$ with generator $(A, D(A))$. Assume that there exists an open connected subset $U$ and weakly analytic functions $f_{j}: U \rightarrow X, j \in J$, such that

(i) $U \cap i \mathbb{R} \neq \emptyset$,

(ii) $f_{j}(\lambda) \in \operatorname{ker}(\lambda I-A)$ for every $\lambda \in U, j \in J$,

(iii) for any $x^{*} \in X^{*}$, if $\left\langle f_{j}(\lambda), x^{*}\right\rangle=0$ for all $\lambda \in U$ and $j \in J$ then $x^{*}=0$.

Then $\left\{T_{t}\right\}_{t \geq 0}$ is Devaney chaotic and topologically mixing.

On the other hand, a $C_{0}$-semigroup is distributionally chaotic if there are an uncountable set $S \subset X$ and $\delta>0$, so that for each $\varepsilon>0$ and each pair $x, y \in S$ of distinct points we have

$$
\begin{gathered}
\overline{\operatorname{Dens}}\left\{s \geq 0:\left\|T_{s} x-T_{s} y\right\| \geq \delta\right\}=1 \text { and } \\
\overline{\operatorname{Dens}}\left\{s \geq 0:\left\|T_{s} x-T_{s} y\right\|<\varepsilon\right\}=1,
\end{gathered}
$$

where $\overline{\operatorname{Dens}}(B)$ is the upper density of a Lebesgue measurable subset $B \subset \mathbb{R}_{0}^{+}$defined as $\lim \sup _{t \rightarrow \infty} \frac{\mu(B \cap[0, t])}{t}$ with $\mu$ standing for the Lebesgue measure on $\mathbb{R}_{0}^{+}$. Distributional chaos is equivalent to the existence of distributional irregular vectors ABMP13, BBMP13. Furthermore, whenever the Desch-Schappacher-Webb criterion can be applied we have that the $C_{0}$-semigroup is not only Devaney chaotic but also distributionally chaotic, see [BBMGP11, Cor. 31] and [BC12, Rem. 3.8].

We refer to the monographs BM09, GEPM11 for the basic theory on chaotic linear dynamics. In particular, [GEPM11, Ch. 7] for the dynamics of $C_{0}$-semigroups of operators.

\subsection{Strong mixing measures}

Some analogous properties to the aforementioned ones can be introduced when the $C_{0}$-semigroup is defined on a probability space $(X, \mathcal{B}, \mu)$, where $X$ is a Banach space and $\mathcal{B}$ denotes the $\sigma$-algebra of Borel subsets of $X$. We say that a Borel probability measure $\mu$ has full support if for any non-empty open set $U \subset X$ we have $\mu(U)>0$. A measure $\mu$ is said to be $T_{t}$-invariant if for all $A \in \mathcal{B}$ we have that $\mu(A)=\mu\left(T_{t}^{-1}(A)\right)$ for all $t \geq 0$. A $C_{0}$-semigroup is strongly mixing if

$$
\lim _{t \rightarrow \infty} \mu\left(A \cap T_{t}^{-1}(B)\right)=\mu(A) \mu(B) \quad \text { for all } A, B \in \mathcal{B} .
$$

We recall that strongly mixing implies ergodicity, i.e. for each $A \in \mathcal{B}$ such that $T^{-1}(A)=A$, either $\mu(A)=0$, or $\mu(A)=1$. 
Very recently it has been stated that the sufficient criterion for frequent hypercyclicity of $C_{0}$-semigroups given by Mangino and Peris in [MP11] also yields the existence of invariant Borel probability measures on $X$ that are strongly mixing and have full support [MAP15]. In [MP11, Cor. 2.3] some conditions, expressed in terms of eigenvector fields for the infinitesimal generator of the $C_{0}$-semigroup, were given which ensure the existence of invariant strongly mixing measures. A different argument for the existence of invariant strongly mixing measures for $C_{0}$-semigroups has been obtained in BM15] under weaker assumptions on the eigenvector fields for the generator.

Theorem 2.2. Let $X$ be a separable complex Banach space and let $\{T\}_{t>0}$ be a $C_{0}$-semigroup on $X$ with generator $A$. Assume that there exists a family $\left(f_{j}\right)_{j \in \Gamma}$ of locally bounded measurable maps $f_{j}: I_{j} \rightarrow X$ such that $I_{j}$ is an interval in $\mathbb{R}, f_{j}\left(I_{j}\right) \subset D(A)$, where $D(A)$ denotes the domain of the generator, $A f_{j}(t)=i t f_{j}(t)$ for every $t \in I_{j}, j \in \Gamma$ and $\operatorname{span}\left\{f_{j}(t): j \in \Gamma, t \in I_{j}\right\}$ is dense in $X$. If either
a) $f_{j} \in C^{2}\left(I_{j}, X\right), j \in \Gamma$,

or

b) $X$ does not contain $c_{0}$ and $\left\langle\varphi, f_{j}\right\rangle \in C^{1}\left(I_{j}\right), \varphi \in X^{\prime}, j \in \Gamma$,

then there is a $\left(T_{t}\right)_{t \geq 0}$-invariant strongly mixing Borel probability measure $\mu$ on $X$ with full support.

We will apply this result to the solution $C_{0}$-semigroup of the Lighthill-Whitham-Richards equation.

\subsection{Spaces of analytic functions of Herzog type}

Let us consider $\rho>0$ and define the space

$$
X_{\rho}=\left\{f: \mathbb{R} \rightarrow \mathbb{C} ; f(x)=\sum_{n=0}^{\infty} \frac{a_{n} \rho^{n}}{n !} x^{n},\left(a_{n}\right)_{n \geq 0} \in c_{0}\right\},
$$

endowed with the norm $\|f\|=\sup _{n>0}\left|a_{n}\right|$, where $c_{0}$ is the Banach space of complex sequences tending to 0 . Then $X_{\rho}$ is a Banach space of analytic functions with a certain growth control. By its definition it is isometrically isomorphic to $c_{0}$.

\section{Main results}

We first express equation 7 as a first order equation on the product of a certain function space with itself. To do this we set $u_{1}=u$ and $u_{2}=\frac{\partial u}{\partial t}$. Then the associated first order equation is

$$
\left\{\begin{array}{l}
\frac{\partial}{\partial t}\left(\begin{array}{l}
u_{1} \\
u_{2}
\end{array}\right)=\left(\begin{array}{cc}
0 & I \\
\frac{\alpha}{\tau} \frac{\partial^{2}}{\partial x^{2}} & -\frac{1}{\tau} I
\end{array}\right)\left(\begin{array}{l}
u_{1} \\
u_{2}
\end{array}\right), \\
\left(\begin{array}{l}
u_{1}(0, x) \\
u_{2}(0, x)
\end{array}\right)=\left(\begin{array}{l}
\varphi_{1}(x) \\
\varphi_{2}(x)
\end{array}\right), \quad x \in \mathbb{R} .
\end{array}\right.
$$

We set

$$
A:=\left(\begin{array}{cc}
0 & I \\
\frac{\alpha}{\tau} \frac{\partial^{2}}{\partial x^{2}} & -\frac{1}{\tau} I
\end{array}\right)
$$

which is easily seen to be an operator on $X:=X_{\rho} \oplus X_{\rho}$, we have that $\left(e^{t A}\right)_{t \geq 0}$ is a $C_{0}$-semigroup on $X$, which is the solution semigroup of (14) on $X$.

Theorem 3.1. There exists $\rho_{0}>0$ be such that the solution semigroup $\left(e^{t A}\right)_{t \geq 0}$ of (14) is mixing, Devaney and distributionally chaotic on $X_{\rho} \oplus X_{\rho}$ for every $\rho \geq \rho_{0}$. 
Proof. Given $\lambda \in \mathbb{C}$, we define

$$
\varphi_{\lambda, a_{0}, b_{0}}(x)=\sum_{n=0}^{\infty} \frac{a_{n} \rho^{2 n} x^{2 n}}{(2 n) !}+\sum_{n=0}^{\infty} \frac{b_{n} \rho^{2 n+1} x^{2 n+1}}{(2 n+1) !}, \quad x \in \mathbb{R} .
$$

The coefficients $a_{n}$ and $b_{n}, n \geq 1$,depending on $\lambda, \rho, a_{0}$ and $b_{0}$ will be determined later. Let $U$ be the open disk of radius 1 centred at zero. If $\lambda \in U$ we consider the functions $f_{a_{0}, b_{0}}: U \rightarrow X$, given by

$$
f_{a_{0}, b_{0}}(\lambda)=\left(\begin{array}{c}
\varphi_{\lambda, a_{0}, b_{0}} \\
\lambda \varphi_{\lambda, a_{0}, b_{0}}
\end{array}\right) .
$$

We will show that, for suitable $\left(a_{n}\right)$ and $\left(b_{n}\right)$,

$$
f_{a_{0}, b_{0}}(\lambda) \in \operatorname{ker}(\lambda I-A), \quad \lambda \in U, a_{0}, b_{0} \in \mathbb{R} .
$$

For convenience, we write $\varphi=\varphi_{\lambda, a_{0}, b_{0}}$. We need to prove that

$$
\lambda^{2} \varphi=-\frac{c}{T} \frac{\partial \varphi}{\partial x}+\frac{D}{T} \frac{\partial^{2} \varphi}{\partial x^{2}}-\frac{\lambda}{T} \varphi,
$$

thus

160

$$
\begin{gathered}
\left(\lambda^{2}+\frac{\lambda}{T}\right) \varphi=-\frac{c}{T}\left(\sum_{n=1}^{\infty} \frac{a_{n} \rho^{2 n} x^{2 n-1}}{(2 n-1) !}+\sum_{n=0}^{\infty} \frac{b_{n} \rho^{2 n+1} x^{2 n}}{(2 n) !}\right) \\
+\frac{D}{T}\left(\sum_{n=1}^{\infty} \frac{a_{n} \rho^{2 n} x^{2 n-2}}{(2 n-2) !}+\sum_{n=1}^{\infty} \frac{b_{n} \rho^{2 n+1} x^{2 n-1}}{(2 n-1) !}\right)
\end{gathered}
$$

$$
=\sum_{n=0}^{\infty} \frac{1}{T}\left(D \rho^{2} a_{n+1}-c \rho b_{n}\right) \frac{\rho^{2 n} x^{2 n}}{(2 n) !}+\sum_{n=0}^{\infty} \frac{1}{T}\left(D \rho^{2} b_{n+1}-c \rho a_{n+1}\right) \frac{\rho^{2 n+1} x^{2 n+1}}{(2 n+1) !} .
$$

A comparison of coefficients yields the following difference equation

$$
\begin{gathered}
\left(\lambda^{2}+\frac{\lambda}{T}\right) a_{n}=\frac{1}{T}\left(D \rho^{2} a_{n+1}-c \rho b_{n}\right) \\
\left(\lambda^{2}+\frac{\lambda}{T}\right) b_{n}=\frac{1}{T}\left(D \rho^{2} b_{n+1}-c \rho a_{n+1}\right)
\end{gathered}
$$

which can be written in a matrix form as

$$
\left(\begin{array}{l}
a_{n+1} \\
b_{n+1}
\end{array}\right)=B(\lambda, \rho)\left(\begin{array}{l}
a_{n} \\
b_{n}
\end{array}\right), \quad n \geq 0
$$

where

$$
B(\lambda, \rho)=\left(\begin{array}{cc}
\frac{\lambda^{2} T+\lambda}{D \rho^{2}} & \frac{c}{D \rho} \\
\frac{c\left(\lambda^{2} T+\lambda\right)}{D^{2} \rho^{3}} & \frac{D \lambda^{2} T+D \lambda+c^{2}}{D^{2} \rho^{2}}
\end{array}\right)
$$

It is clear that there exists $\rho_{0}>0$ such that, for $\rho \geq \rho_{0}$, the eigenvalues of $B(\lambda, \rho)$ are of modulus strictly less than 1 , so that the sequences $\left(a_{n}\right)$ and $\left(b_{n}\right)$ tend to 0 . This means that $\varphi \in X_{\rho}$.

According to Theorem 2.1 it remains to prove that, for any $x^{*} \in X_{\rho}^{*} \oplus X_{\rho}^{*}$, the functions $\lambda \rightarrow$ $\left\langle f_{z_{0}, z_{1}}(\lambda), x^{*}\right\rangle, z_{0}, z_{1} \in \mathbb{R}$, are holomorphic on $U$, and that it if they all vanish on $U$ then $x^{*}=0$.

Thus, let $x^{*} \in X_{\rho}^{*} \oplus X_{\rho}^{*}$. By the isomorphism of $X_{\rho}$ with $c_{0}, x^{*}$ can be represented in a canonical way by a pair $\left(\left(c_{n}\right)_{n \geq 0},\left(d_{n}\right)_{n \geq 0}\right) \in \ell^{1} \oplus \ell^{1}$. We then have that, for $\lambda \in U$,

$$
\begin{aligned}
& \left\langle f_{a_{0}, b_{0}}(\lambda), x^{*}\right\rangle= \\
& \sum_{n=0}^{\infty} a_{n}(\lambda) c_{2 n}+\sum_{n=0}^{\infty} b_{n}(\lambda) c_{2 n+1}+\lambda \sum_{n=0}^{\infty} a_{n}(\lambda) d_{2 n}+\lambda \sum_{n=0}^{\infty} b_{n}(\lambda) d_{2 n+1} .
\end{aligned}
$$


Since these series converge uniformly on $U$, and each $a_{n}(\lambda)$ and $b_{n}(\lambda)$ is a polynomial on $\lambda$, this implies that each function $f_{a_{0}, b_{0}}$ is weakly holomorphic on $U$.

Finally, suppose that all the functions $\lambda \rightarrow\left\langle f_{a_{0}, b_{0}}(\lambda), x^{*}\right\rangle, a_{0}, b_{0} \in \mathbb{R}$, vanish on $U$. Proceeding inductively we deduce that $c_{n}=d_{n}=0$ for all $n \geq 0$, and hence that $x^{*}=0$.

Since holomorphic functions on an open set that intersects the imaginary axis are certainly $C^{\infty}$ when restricted to this intersection, the argument of the previous result yields the hypothesis of Theorem 2.2 . which turns out on the existence of strongly mixing measures.

Corollary 1. There exists $\rho_{0}>0$ be such that the solution semigroup $\left(e^{t A}\right)_{t \geq 0}$ of (14) admits an invariant strongly mixing probability measure with full support on $X_{\rho} \oplus X_{\rho}$ for every $\rho \geq \rho_{0}$.

\section{Acknowledgements}

The authors are supported by MEC Project MTM2013-47093-P. The second and third authors are supported by GVA, Project PROMETEOII/2013/013.

\section{References}

[ABMP13] Angela A. Albanese, Xavier Barrachina, Elisabetta M. Mangino, and Alfredo Peris. Distributional chaos for strongly continuous semigroups of operators. Commun. Pure Appl. Anal., 12(5):2069-2082, 2013.

[AP12] Javier Aroza and Alfred Peris. Chaotic behaviour of birth-and-death models with proliferation. J. Difference Equ. Appl., 18(4):647-655, 2012.

[BBCP05] Teresa Bermúdez, Antonio Bonilla, José A. Conejero, and Alfredo Peris. Hypercyclic, topologically mixing and chaotic semigroups on Banach spaces. Studia Math., 170(1):57-75, 2005.

[BBMGP11] T. Bermúdez, A. Bonilla, F. Martínez-Giménez, and A. Peris. Li-Yorke and distributionally chaotic operators. J. Math. Anal. Appl., 373(1):83-93, 2011.

[BBMP13] N. C. Bernardes, Jr., A. Bonilla, V. Müller, and A. Peris. Distributional chaos for linear operators. J. Funct. Anal., 265(9):2143-2163, 2013

[BC12] Xavier Barrachina and J. Alberto Conejero. Devaney chaos and distributional chaos in the solution of certain partial differential equations. Abstr. Appl. Anal., pages Art. ID 457019, 11, 2012.

[BCMASS15] Xavier Barrachina, J. A. Conejero, Marina Murillo-Arcila, and Juan B. Seoane-Sepúlveda. Distributional chaos for the forward and backward control traffic model. Preprint, 2015.

[BL01] Jacek Banasiak and Mirosław Lachowicz. Chaos for a class of linear kinetic models. C. R. Acad. Sci. Paris Série II, 329:439-444, 2001.

[BL02] Jacek Banasiak and Mirosław Lachowicz. Topological chaos for birth-and-death-type models with proliferation. Math. Models Methods Appl. Sci., 12(6):755-775, 2002.

[BM99] Mark Brackstone and Mike McDonald. Car-following: a historical review. Transportation Research Part F: Traffic Psychology and Behaviour, 2(4):181 - 196, 1999.

[BM05] Jacek Banasiak and Marcin Moszyński. A generalization of Desch-Schappacher-Webb criteria for chaos. Discrete Contin. Dyn. Syst., 12(5):959-972, 2005.

[BM09] Frédéric Bayart and Étienne Matheron. Dynamics of linear operators, volume 179 of Cambridge Tracts in Mathematics. Cambridge University Press, Cambridge, 2009.

[BM11] Jacek Banasiak and Marcin Moszyński. Dynamics of birth-and-death processes with proliferation—stability and chaos. Discrete Contin. Dyn. Syst., 29(1):67-79, 2011.

[BM15] Frédéric Bayart and Étienne Matheron. Mixing operators and small subsets of the circle. Ergodic Theory Dynam. Systems, page To appear., 2015.

[CLR15] J. A. Conejero, C. Lizama, and F. Rodenas. Chaotic behaviour of the solutions of the Moore-Gibson-Thompson equation. Applied Math. \& Inf. Sciences, 9(5):1-6, 2015.

[CM10] José A. Conejero and Elisabetta M. Mangino. Hypercyclic semigroups generated by Ornstein-Uhlenbeck operators. Mediterr. J. Math., 7(1):101-109, 2010.

[CMASS15] J. A. Conejero, Marina Murillo-Arcila, and Juan B. Seoane-Sepúlveda. Linear chaos for the quick-thinking-driver model. Semigroup Forum, page To appear., 2015.

[CPT10] J. A. Conejero, A. Peris, and M. Trujillo. Chaotic asymptotic behavior of the hyperbolic heat transfer equation solutions. Internat. J. Bifur. Chaos Appl. Sci. Engrg., 20(9):2943-2947, 2010.

[CRT15] J. Alberto Conejero, Francisco Rodenas, and Macarena Trujillo. Chaos for the Hyperbolic Bioheat Equation. Discrete Contin. Dyn. Syst., 35(2):653-668, 2015.

[DSW97] Wolfgang Desch, Wilhelm Schappacher, and Glenn F. Webb. Hypercyclic and chaotic semigroups of linear operators. Ergodic Theory Dynam. Systems, 17(4):793-819, 1997. 
[EN00] Klaus-Jochen Engel and Rainer Nagel. One-parameter semigroups for linear evolution equations, volume 194 of Graduate Texts in Mathematics. Springer-Verlag, New York, 2000. With contributions by S. Brendle, M. Campiti, T. Hahn, G. Metafune, G. Nickel, D. Pallara, C. Perazzoli, A. Rhandi, S. Romanelli and R. Schnaubelt.

[GEPM11] Karl-G. Grosse-Erdmann and Alfredo Peris Manguillot. Linear chaos. Universitext. Springer, London, 2011.

[Her97] Gerd Herzog. On a universality of the heat equation. Math. Nachr., 188:169-171, 1997.

[LG04] Keping Li and Ziyou Gao. Nonlinear dynamics analysis of traffic time series. Modern Physics Letters B, 18(26n27):1395-1402, 2004.

[Li05] Tong Li. Nonlinear dynamics of traffic jams. Physica D: Nonlinear Phenomena, 207(1-2):41 - 51, 2005.

[Lus10] C. Lustri. Continuum modelling of traffic flow. Special topic report, Oxford University, 2010.

[LW55] M. J. Lighthill and G. B. Whitham. On kinematic waves. II. A theory of traffic flow on long crowded roads. Proc. Roy. Soc. London. Ser. A., 229:317-345, 1955.

[MAP15] M. Murillo-Arcila and A. Peris. Strong mixing measures for $C_{0}$-semigroups. Rev. R. Acad. Cienc. Exactas Fís. Nat. Ser. A Math. RACSAM, 109(1):101-115, 2015.

[MDM05] Sven Maerivoet and Bart De Moor. Cellular automata models of road traffic. Phys. Rep., 419(1):1-64, 2005

[MP11] Elisabetta M. Mangino and Alfredo Peris. Frequently hypercyclic semigroups. Studia Math., 202(3):227-242, 2011.

[PA92] V. Protopopescu and Y. Y. Azmy. Topological chaos for a class of linear models. Math. Models Methods Appl. Sci., 2(1):79-90, 1992.

[Paz83] A. Pazy. Semigroups of linear operators and applications to partial differential equations, volume 44 of Applied Mathematical Sciences. Springer-Verlag, New York, 1983.

[Ric56] Paul I. Richards. Shock waves on the highway. Operations Res., 4:42-51, 1956. 